Philosophie ANTIQUE
Philosophie antique

Problèmes, Renaissances, Usages

$18 \mid 2018$

L'athéisme antique

\title{
Les athéismes de Bion de Borysthène
}

\section{Suzanne Husson}

\section{(2) OpenEdition}

\section{Journals}

Édition électronique

URL : https://journals.openedition.org/philosant/1045

DOI : 10.4000/philosant.1045

ISSN : 2648-2789

\section{Éditeur}

Éditions Vrin

\section{Édition imprimée}

Date de publication : 1 novembre 2018

Pagination : 193-215

ISBN : 978-2-7574-2372-1

ISSN : $1634-4561$

\section{Référence électronique}

Suzanne Husson, « Les athéismes de Bion de Borysthène », Philosophie antique [En ligne], 18 | 2018, mis en ligne le 01 novembre 2019, consulté le 09 décembre 2022. URL : http://

journals.openedition.org/philosant/1045; DOI : https://doi.org/10.4000/philosant.1045

\section{$@ \oplus \Theta \Theta$}

Creative Commons - Attribution - Pas d'Utilisation Commerciale - Pas de Modification 4.0 International - CC BY-NC-ND 4.0

https://creativecommons.org/licenses/by-nc-nd/4.0/ 


\section{LES ATHÉISMES DE BION DE BORYSTHÈNE}

Suzanne Husson

Sorbonne Université, Centre Léon Robin (UMR 8061), Labex RESMED

RÉSUMÉ. Bion de Borysthène, qui a fréquenté l'école académicienne (Xénocrate), cynique (Cratès) et cyrénaïque (Théodore), ne faisait pas partie des listes traditionnelles d'athées en circulation dans l'Antiquité, mais on lui attribue pourtant la formule athée d'après laquelle « il n'y a pas de dieux » (Diogène Laërce, IV, 55). L'examen des témoignages montre qu'il pouvait être qualifié d'athée à deux niveaux. Tout d'abord, aussi bien au niveau théorique que pratique, il adoptait une attitude très critique à l'égard des pratiques religieuses et des récits mythiques traditionnels dont il mettait en valeur les contradictions et plus largement contestait la partition du réel entre sacré et profane (Sénèque, Des bienfaits, VII 7, 1-2). Par ailleurs, Bion s'est également opposé à la théologie philosophique épicurienne et, en particulier, à son anthropomorphisme. Il s'appuyait, entre autres, sur une thèse (« chaque genre de vivants a une forme particulière dans son genre particulier », Démétrius Lacon, De la forme du dieu, XIX, 13), compatible avec l'épicurisme. Pour cette raison, Démétrius Lacon entreprit d'y répondre en montrant que cette prémisse, pouvant aussi servir à contester les théologies astrales (platoniciennes ou stoïciennes), ne vaut que pour les êtres accessibles par la sensation, ce qui n'est pas le cas des dieux. Cette polémique, dont l'enjeu est la limite de l'application du principe d'isonomie, a laissé des traces jusque dans le De signis de Philodème.

Summary. Bio of Borysthenes, who attended the Academy (Xenocrates) and also the Cynic (Crates) and Cyrenaic schools (Theodorus) was not included in the atheists' traditional lists in circulation in Antiquity, but the atheistic formula according to which "there are no gods" (D. L. IV, 55) is attributed to him. The review of the fragments shows that he could be qualified as atheist on two levels. First of all, he adopted a very critical approach towards the religious practices and the traditional mythical narratives, by highlighting their contradictions and more widely he disputed the division of the reality between sacred and profane (Seneca, De Beneficiis, VII 7, 1-2). Besides, Bio also opposed the philosophic epicurean theology and, particularly, its anthropomorphism. He

Philosophie antique, ${ }^{\circ} 18$ (2018), 193-215 
relied, among others, on a proposition ("every kind of living being has a particular shape in itsparticular kind", Demetrius Laco, On the shape of the god, XIX, 13), compatible with the Epicureanism. Accordingly, Demetrius Laco undertook to answer by showing that this premise, which is also able to refute the astral theologies (Platonists or Stoics), applies only to the beings accessible through the senses, which is not the case of the gods. This debate, whose issue is the limit of the application of the isonomy principle, left its mark in Philodemus De Signis. 
Bion de Borysthène, qui vécut approximativement entre 335 et 245 av. J.-C. ${ }^{1}$, ne faisait pas partie des listes traditionnelles d'athées telles qu'elles nous sont parvenues, c'est-à-dire des personnages censés avoir nié l'existence des dieux ${ }^{2}$; c'est pourtant l'un des premiers exemples documentés, sinon le premier, d'athée se repentant sur son lit de mort - ce que Diogène Laërce, friand de ce genre d'anecdotes, n'a pas manqué de rapporter :

Dans la conversation, il tenait souvent des propos très irrespectueux envers

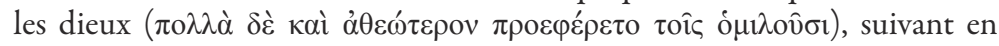
cela l'exemple de Théodore. Par la suite, lorsqu' il fut atteint par la maladie, comme le racontèrent les gens de Chalcis - c'est là en effet qu'il mourut -, il

1. Notre connaissance de la biographie de Bion dérive essentiellement de Diogène Laërce (D. L.) IV 46-58 qui, lui-même, cite comme sources le stoïcien Persée et un certain Philonidès (DL IV 47). Il serait arrivé à Athènes avant la mort de Xénocrate et serait mort avant Antigone Gonatas, ce qui permet de le situer dans le temps de façon vraisemblable ( $c f$. Kindstrand 1976, p. 5-6 et 106-107 pour les fragments biographiques).

2. La première liste connue est celle qu'Épicure avait dressée dans son traité Sur la nature, au livre 12, et que Philodème rapporte dans son Sur la piété (col. XIX, l. 1-15; éd. Obbink) [cf. Bremmer 2007 p. 19-20], en disant qu'elle comprenait Prodicos, Diagoras et Critias, mais que ce n'étaient pas les seuls personnages cités car il y en avait d'autres ( $\kappa \alpha ̆[\lambda \lambda o r \varsigma, 1.8)$, de sorte que nous ne pouvons pas savoir si éventuellement Bion, un contemporain, s'y trouvait. La seconde liste nous vient de Clitomaque ( $c f$. Winiarczyk 1976), elle est reprise par Sextus Empiricus (M. IX 51-59) et comprend, dans leur ordre de citation, Évhémère, Diagoras, Prodicos, Théodore, Critias et, selon certains, Protagoras et Épicure. Cependant, nous ne pouvons pas savoir quels autres auteurs y étaient inclus, car Sextus, dans un premier temps, signale que les athées sont Évhémère, Diagoras, Prodicos et ő’ $\lambda \lambda$ or $\pi \alpha \mu \pi \lambda \eta \theta \varepsilon i \hat{\iota}$, « une multitude d'autres », expression qui correspond difficilement aux seuls autres auteurs ajoutés dans la suite (à savoir, Théodore et Critias et, avec réserve, Protagoras et Épicure). D'autre part, si Théodore, l'un des maîtres de Bion, s'y trouvait, il n'est pas invraisemblable que le disciple s'y trouvât. Aussi A. B. Drachmann 1922 p. 18, malgré l'absence de Bion dans les listes traditionnelles d'athées, le classe néanmoins parmi ceux qui ont été qualifiés d'atheoi. 
se laissa convaincre d'accepter des amulettes et de se repentir des injures qu' il avait lancées contre la divinité 3 .

L'ironie est que ce personnage mourra, non seulement en se repentant de son impiété - mal parler des dieux, en effet, est une impiété - mais en adoptant des pratiques populaires extravagantes pour un philosophe (le port d'amulettes) relevant de la superstition, c'est-à-dire de la deisidaimonia, par opposition à la véritable piété (eusebeia) ${ }^{4}$. En effet, juste après avoir développé ce récit, Diogène Laërce tournera une épigramme, dont le sel n'échappera pas, par exemple, à Montaigne ${ }^{5}$ :

Nous avons entendu dire que Bion, qu' a fait naître la terre scythe de Borysthène disait que les dieux ne sont rien en vérité.

S'il était resté attaché à cette opinion, il aurait été normal de dire :

« Il a pensé comme il voulait; mal, mais c'était son avis ».

En vérité, une fois affligé d'une longue maladie et craignant de mourir, lui qui niait l'existence des dieux ${ }^{6}$, qui n'avait jamais visité un temple,

(56) qui accablait de sarcasmes les mortels qui sacrifiaient aux dieux, non seulement il a rempli, sur le foyer, sur les autels, sur la table, les narines des dieux avec l'odeur (des victimes), avec leur graisse, avec l'encens, non seulement il a dit : «J'ai péché, pardonnez-moi mes fautes passées », mais sans réticence il tendit son cou à une vieille femme pour un charme et il se laissa convaincre d'attacher autour de ses bras des brassards de cuir, (57) et il posa au-dessus de sa porte un nerprun et une branche de laurier, prêt à tout subir plutôt que la mort.

Sot qui voulait, en échange d'une modeste offrande, que la divinité existe, comme si les dieux existaient quand il plaisait à Bion de le croire ${ }^{7}$.

Eh bien, recouvrant en vain la raison, lorsqu'il n'était plus que du charbon morveux,

il tendit la main en disant : « Salut, Pluton, salut ! »

(DL IV, 55-57, trad. T. Dorandi)

L'anecdote elle-même décrit l'attitude de Bion comme á $\theta \varepsilon \omega ́ \tau \varepsilon \rho o v$ (DL IV, 54), mais il ne s'agit ici que de l'athéisme pratique de l'impie refusant le respect dû aux dieux. Cependant, le doxographe dans son épigramme va plus loin et fait de Bion un athée théorique affirmant que les dieux

3. DL IV 54 (= F1A Kindstrand 1976), trad. T. Dorandi (dans M.-O. Goulet-Cazé (dir.) 1999), légèrement modifiée.

4. Opposition constituée par Théophraste dans sa description du «Superstitieux » (Caractères, 16), cf. Martin 2004 p. 21-35.

5. Comme le montre le résumé qu'il en fait : « Ils récitent [i.e. on raconte] de Bion, qu'infect des atheismes de Theodorus, il avoit esté longtemps se moquant des hommes religieux : mais, la mort le surprenant, qu' il se rendit aux plus extremes superstitions, comme si les Dieux s'ostoyent et se remettoyent selon l'affaire de Bion. » Cf. Essais, II, 12, « Apologie de Raymond Sebon, » p. 446, (éd. Villey 1988).

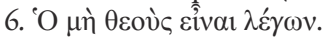

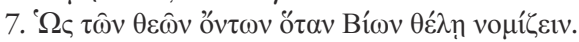


n'existent pas (DL IV, 55), sans doute parce qu'il fut l'élève de Théodore de Cyrène, connu comme athée depuis, au moins, la liste de Clitomaque ${ }^{8}$. Comme à Théodore, on attribue donc à Bion la position d'après laquelle « il n'y a pas de dieux », mais alors que le cyrénaïque l'aurait soutenue dans un Peri theon ${ }^{9}$, Bion aurait été plus prudent, ce qui expliquerait peut-être que, contrairement à son maître, il n'ait pas été inquiété ${ }^{10}$ :

En tout cas Cratès lui ayant demandé si les dieux se réjouissent des génuflexions et des prières, on dit que Stilpon fit cette réponse : « Ne m'interroge pas là-dessus en pleine rue, insensé que tu es, mais seul à seul ». C'est la même réponse que fit Bion quand on lui demanda si les dieux existent ( $\tau$ ò

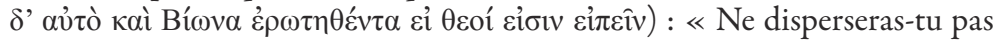
loin de moi la foule, calamiteux vieillard ? » (DL II, $117=$ F25 Kindstrand, trad. M.-O. Goulet-Cazé)

La tradition a donc retenu ce personnage ambigu d'un athée n'assumant pas son athéisme avec parrhesia et auquel, en fin de compte, manque le courage par lequel le véritable philosophe manifeste la vérité, telle qu'il la comprend, par sa vie ${ }^{11}$. Mais, au-delà de cette étiquette doxographique, quel style d'athée Bion pouvait-il être ? Qu'est-ce qui, par rapport à ce que nous savons par ailleurs de ses positions, pouvait justifier son athéisme ? Aux difficultés méthodologiques habituelles liées à l'approche de l'athéisme dans l'Antiquité s'ajoute le fait que Bion apparaît comme un penseur difficile à cerner.

En effet, la question de savoir dans quelle école il faut le situer, et, en particulier, si on peut le classer parmi les cyniques, est encore débattue. Comme nous l'avons vu, il fréquenta l'école cyrénaïque ; d'autre part Diogène Laërce ne le présente pas dans le livre VI, consacré au cynisme, mais dans le livre IV, consacré aux successeurs de Platon, entre Arcésilas et Carnéade, donc parmi les représentants de la moyenne Académie.

Le parcours philosophique de Bion, en effet, témoigne de sa curiosité : arrivé avant 314 à Athènes, il rencontre Xénocrate ${ }^{12}$, et commence donc par fréquenter l'Académie où il suit son enseignement, puis vraisemblablement celui de Polémon qui lui succède immédiatement, ainsi qu'en parallèle celui de Cratès d'Athènes ${ }^{13}$.

Diogène Laërce (IV 51) nous dit qu'il adopta ensuite le mode de vie

8. Cf. supra n. 2, et fr. 28-44 Winiarczyk 1981a et Socratis et Socraticorum reliquiae (= SSR) IV H 13-25, Giannantoni 1990 vol. 2.

9. DL II, 97 (= fr. 45 Winiarczyk 1981a, SSR IV H 13) et Sextus Empiricus, M. IX, 55 (= fr. 46 Winiarczyk 1981a, SSR IV H 23).

10. Sur le procès pour impiété et l'exil de Théodore, voir Winiarczyk 1981b p. 67-69.

11. Cf. Foucault 2009.

12. Si nous en croyons une anecdote (DL IV, $51=$ T22 Kindstrand 1976), 314 est la date de la mort de Xénocrate (Kindstrand 1976 p. 5, 56-57 ; Navia 1996 p. 152).

13. Cf. Kindstrand 1976 p. 10. 


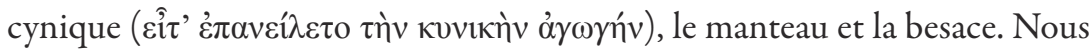
pouvons en déduire qu'il fut vraisemblablement l'élève de Cratès de Thèbes, alors représentant de l'enseignement de Diogène de Sinope à Athènes, et qu'il eut sans doute comme condisciple Zénon de Citium, élève de Cratès lui aussi, et fondateur du stoïcisme ${ }^{14}$.

Enfin il suivit les leçons de Théodore de Cyrène, dit « l'athée », ainsi que celles du péripatéticien Théophraste. Si la tradition antique ne semble donc pas l'avoir formellement identifié comme un cynique ${ }^{15}$, il n'en reste pas moins que l'on retrouve dans les témoignages qu'elle nous a conservés de lui beaucoup de traits cyniques $^{16}$ : pouvons-nous alors dire que son athéisme est dû uniquement à l'influence théodoréenne (comme le suggère Diogène Laërce) ou bien relève-t-il également d'un certain héritage cynique, ambivalent lui aussi ?

La question du rapport entre cynisme et athéisme est, en effet, difficile à trancher. Si nous prenons l'exemple de Diogène de Sinope, nous constatons qu'il apparaît comme un pourfendeur en acte des pratiques religieuses traditionnelles : il refusait de se faire initier aux Mystères et sa provocation serait allée, si l'on en croit la tradition, jusqu'à troubler activement les manifestations de piété ${ }^{17}$. D'autre part sont attribuées à Diogène des réparties qui le sont également à des athées notoires comme Diagoras de Mélos et Théodore $^{18}$. Diogène Laërce rapporte ainsi une dénonciation intéressante du biais de confirmation :

Comme quelqu'un s'étonnait devant les ex-voto de Samothrace, « il y en aurait beaucoup plus », dit-il, « si les gens qui n’ont pas été sauvés en avaient offert aussi ». D'autres rapportent le mot à Diagoras de Mélos. (DL VI, $59=$ SSR VB 342, trad. M.-O. Goulet-Cazé)

Les propos de Diogène sont donc apparus à certains comme assimilables à ceux d'un athée célèbre. Cependant la tradition n'a pas retenu pour lui le qualificatif $d^{\prime} \alpha{ }^{\prime} \theta \varepsilon \circ \varsigma^{19}$. La raison en est sans doute dans le fait que, par ailleurs,

14. Cf. Kindstrand 1976 p. 10-11; Navia 1996.

15. Sauf Polystrate dans ses attaques contre l'école cynique ( $c f$. T 27 Kindstrand 1976).

16. Dans les Diatribes de Télès en particulier, qui sont avec Diogène Laërce la source principale des fragments de Bion (cf. Kindstrand 1976 p. 82-86, Fuentes González 1998 p. 23-32).

17. Par exemple : « Il [Diogène] vit un jour une femme qui se prosternait devant les dieux dans une attitude particulièrement indécente. Voulant la débarrasser de sa superstition, à ce qui dit Zoïlos de Pergée, il s'approcha d'elle et dit : "Ne crains-tu pas, femme, que si un jour un dieu se tient derrière toi, - car tout est rempli de la divinité -, ton attitude ne soit indécente ?"” », DL VI, 37 (= SSR V B 344), trad. M.-O. Goulet Cazé 1999.

18. Cf. SSR V B 131, 342, 103, 334; Giannantoni 1990 vol. IV p. 547-550, et Winiarczyck 1976b, 1981c.

19. Il n'est accusé d'athéisme que dans une chrie, attribuée également par ailleurs à Théodore (DL II, 102) : « À Lysias l'apothicaire qui lui demandait s'il croyait à l'existence des

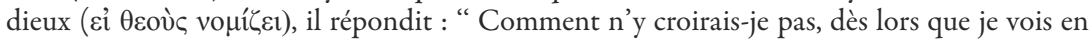
toi un ennemi des dieux ?'”. D'autres attribuent le trait à Théodore » (DL VI, 42, trad. M.-O. 
le cynique se référait aux dieux dans son enseignement, notamment en tant que paradigmes moraux, par exemple dans la formule : « le propre des dieux est de ne manquer de rien, celui de ceux qui sont semblables aux dieux, de manquer de peu de choses » (DL VI, 105 = SSR V B 135), de sorte qu'un dévot comme Julien avait pu le présenter comme un exemple de piété20. Les premiers cyniques apparaissaient donc au niveau pratique comme des athées, refusant de suivre les coutumes religieuses, voire les critiquant ouvertement, mais leur enseignement moral ne permettait pas de les présenter comme des athées théoriques ${ }^{21}$.

À ces difficultés propres au personnage de Bion et à ceux qui l'ont influencé, s'ajoute la question classique de l'imprécision du concept d'athéisme ${ }^{22}$ appliqué aux figures de l'Antiquité, qui nous oblige à distinguer plusieurs niveaux d'analyse ; nous en distinguerons pour notre part trois ${ }^{23}$.

Le premier niveau est le niveau pratique : ont pu être qualifiés d'atheoi ceux qui commettaient des actes d'impiété ou refusaient les pratiques de la religion traditionnelle en vigueur dans la cité. Les deux autres niveaux sont d'ordre théorique : on pouvait, en effet, qualifier d'athées ceux qui mettaient

Goulet-Cazé 1999). Winiarczyck 1981c p. 38 estime que la chrie remontait à Théodore et ne fut attribuée à Diogène que dans un deuxième temps, voir aussi A. B. Drachman 1922 p. 109. Quoi qu' il en soit, Diogène n'est pas ici présenté comme un penseur ouvertement athée, bien que cette réponse ad hominem reste foncièrement ambiguë.

20. Cf. Julien Empereur, Discours, VII, 7-9; IX [VI], 17.

21. Nous n'envisageons pas ici le cas d'Antisthène, qu'une partie de la tradition présente comme le fondateur du cynisme : il n'avait pas, au niveau pratique, la même attitude d'abstention religieuse (il s'était par exemple fait initier aux Mystères, $c f$. DL VI, 4) et soutenait une théologie explicitement monothéiste. Quant aux cyniques proprement dits, c'est-à-dire d'inspiration diogénienne, il faut considérer de plus qu'ils refusaient les spéculations théoriques, de sorte qu' ils ne pouvaient pas s'engager explicitement dans une physique, et encore moins une métaphysique, qui leur aurait permis de développer une théologie rationnelle justifiant leurs références éthiques aux dieux. Ce désinvestissement de la réflexion théologique a conduit M.-O. Goulet-Cazé 1993 à les qualifier d'agnostiques.

22. L'athéisme est en soi un concept qui n'a rien d'une évidence. Pour qu'un athéisme clos et définitif puisse se construire, il faudrait qu'il fût capable de définir un concept universel de la divinité de sorte qu'il puisse s'opposer non seulement à toutes les divinités présentes dans la culture ou la philosophie d'une époque, mais à toute forme de divinité possible. Et même si l'on considère que l'athéisme philosophique consiste à s'opposer à toutes les formes de divinité connues, nous pouvons signaler à quel point la tâche était intellectuellement plus ardue dans l'Antiquité, où les dieux étaient évidemment plus divers, mais aussi les théologies plus variées et non définies, alors que dans un contexte monothéiste le penseur athée n'a, par définition, qu'un seul objet de réflexion, de plus déjà construit au niveau conceptuel par les institutions chargées de définir une orthodoxie religieuse.

23. Sorel 2015 p. 64-65, distingue trois sens du terme ớ $\theta \varepsilon 0 \varsigma$, l'athéisme pratique, c'est-àdire l'impiété, l'athéisme théorique (dire ou penser que les dieux n'existent pas) et être ő $\theta \varepsilon o \varsigma$ au sens d' «être abandonné des dieux », sens qui ici n'est évidemment pas pertinent. 
en doute (Protagoras) ou niaient l'existence des dieux (Théodore) ${ }^{24}$. Mais « penser qu'il n'y a pas de dieux » peut signifier soit que l'on conteste les dieux de la religion traditionnelle, soit l'existence des dieux en général, c'està-dire, à l'époque hellénistique, l'existence des dieux issus des théologies philosophiques, à savoir le monde et les astres (pour Platon et les stoïciens), les dieux métaphysiques des platoniciens (le démiurge dans ses diverses interprétations) et des péripatéticiens (le premier moteur), ainsi que les dieux atomistes de la théologie épicurienne.

Nous pouvons donc ainsi distinguer (1) l'athéisme pratique dans lequel, en plus du comportement extérieur impie, nous incluons les justifications qui peuvent en être données, dans la mesure où elles ne remettent pas en cause l'existence des dieux ${ }^{25}$; (2) l'athéisme théorique dirigé contre les dieux de la religion en vigueur ${ }^{26} ;(3)$ l'athéisme théorique dirigé contre les dieux des philosophes.

Dans le cadre de ces distinctions, jusqu'où Bion peut-il être considéré comme athée ? Au niveau pratique, au-delà de sa propension au blasphème rapportée par Diogène Laërce, nous retrouvons chez lui une critique des pratiques religieuses qui vont, pour certaines, jusqu' à s'attaquer aux croyances communément partagées. Si l'on étend la réflexion à des discussions adressées à des conceptions philosophiques du divin, pouvons-nous cependant considérer qu'elles visent une négation de l'existence des dieux ?

\section{Contre les traditions religieuses : de l'impiété à l'athéisme}

Tout d'abord, nous trouvons chez Bion ce que nous pourrions appeler une critique du sacré tel qu'il peut être défini dans les cadres de la piété traditionnelle de son temps. Le sacré distingue dans l'espace - et, en général, dans les êtres - ce qui appartient au divin, dont on ne peut user sans respecter certains rites et interdits, et ce qui est de l'ordre de la réalité profane vis-à-vis de laquelle les hommes peuvent agir sans précautions supplémentaires ${ }^{27}$.

24. Philodème (PHerc. 1428 col. XIV 32 - XV 8) ainsi distinguait trois raisons qui justifiaient la qualification d'athée : l'agnosticisme à la manière de Protagoras, l'affirmation de la thèse d'après laquelle les dieux n'existent pas, et le fait que cette thèse soit clairement impliquée (cas qui permettait d'accuser Épicure d'athéisme), cf. Bremmer 2007 p. 12.

25. Le comportement impie peut, en effet, être le fait de tenants de l'existence des dieux : sans parler des chrétiens qui, à cause de leur refus du culte en vigueur, ont été qualifiés d'athées, nous trouvons chez Xénophon (Mémorables, I, IV 10) le cas d'Aristodème s'abstenant de toute pratique religieuse parce qu'il estimait que les dieux n'avaient pas besoin de sa $\theta \varepsilon \rho \alpha \pi \varepsilon i ́ \alpha$, de son culte ( $c f$. Sorel 2015 p. 64).

26. Celui-ci peut se présenter comme un niveau supplémentaire de justification théorique de l'athéisme pratique, mais il peut être tout à fait détaché de lui comme chez Épicure, qui, tout en contestant comme impie la représentation commune des dieux, recommandait de respecter, dans un autre esprit, les rites traditionnels ( $c f$. Festugière 1946 chap. IV).

27. Le sacré est si radicalement distingué du profane que les deux domaines ne doivent pas entrer en contact : $c f$. Durkheim 1990 [1912] I, I, 3, p. 55 : « L'opposition de ces deux 
Ainsi Sénèque rapporte que :

Bion a des arguments pour démontrer tantôt que tout le monde est sacrilège, tantôt que personne ne l'est. Lorsqu'il veut précipiter tout le monde du haut de la Roche ${ }^{28}$, il dit :

Quiconque a enlevé ou détruit ou même fait servir à son usage (sustulit et consumpsit, atque in usum suum uertit) ce qui appartient aux dieux est sacrilège ;

or tout appartient aux dieux ;

donc enlever quelque chose, c'est toujours enlever ce qui est aux dieux, à qui tout appartient ;

donc enlever quelque chose, c'est toujours être sacrilège.

Après cela, lorsqu'il veut qu'on force les temples et que l'on pille impunément le Capitole, il prétend qu'il n'y a point d'homme sacrilège parce que tout objet enlevé ne fait que passer de l'endroit qui était aux dieux à l'endroit... qui est aux dieux aussi ${ }^{29}$ !

La première partie de l'argument montre qu'enlever quoi que ce soit de son lieu d'origine est un acte sacrilège. Nous pouvons aller jusqu'à interpréter cette inférence comme une contestation de la notion même de propriété. Être propriétaire, en effet, c'est avoir un droit d'usage sur la chose, un droit de la déplacer voire de la détruire. Or, si déplacer une chose est un acte sacrilège, alors c'est la propriété même qui le devient. Ce ne sont pas seulement les pilleurs de temples qui sont sacrilèges, mais, dans la mesure où nous usons des choses, nous le sommes tous. Nous pouvons voir dans un tel raisonnement une forte inspiration cynique. Non seulement parce que Diogène, dans sa République, abolissait la notion même de propriété privée dans la cité des sages $^{30}$, mais parce qu'il utilisait la formule d'après laquelle « tout appartient aux dieux » comme prémisse dans le syllogisme qui montrait que le sage jouit du même droit de propriété que les dieux : « Tout appartient aux

genres vient, d'ailleurs, se traduire au-dehors par un signe visible qui permet de reconnaître aisément cette classification très spéciale, partout où elle existe. Parce que la notion du sacré est, dans la pensée des hommes, toujours et partout séparée de la notion du profane, parce que nous concevons entre elles une sorte de vide logique, l'esprit répugne invinciblement à ce que les choses correspondantes soient confondues ou simplement mises en contact; car une telle promiscuité ou même une contiguiité trop directe contredisent trop violemment l'état de dissociation où se trouvent ces idées dans les consciences. La chose sacrée, c'est, par excellence, celle que le profane ne doit pas, ne peut pas impunément toucher ».

28. La roche Tarpéienne, d'où les condamnés à mort étaient précipités.

29. Sénèque, Des bienfaits, VII, 7, 1-2 (= F33 Kindstrand 1976), trad. F. Préchac 1927, très légèrement modifiée : (1) Bion modo omnes sacrilegos argumentis esse colligit, modo neminem. Quum omnes de saxo deiecturus est, dicit: Quisquis id quod deorum est, sustulit et consumpsit, atque in usum suum uertit, sacrilegus est: omnia autem deorum sunt: quod quisque ergo tollit, deorum tollit, quorum omnia sunt : ergo quisquis tollit aliquid, sacrilegus est. (2) Deinde quum effringi templa, et expilari impune Capitolium iubet, dicit : Nullum sacrilegium esse; quia quidquid sublatum est ex eo loco qui deorum erat, in eum transfertur locum, qui deorum est.

30. Cf. Husson 2011 p. 106-109. 
dieux ; les dieux sont amis des sages ; tout est commun entre amis ; donc tout appartient aux sages ${ }^{31}$. »

Sénèque, conscient du caractère corrosif de ces propos, fait suivre sa citation de Bion d'une réponse distinguant la propriété selon la nature de la propriété institutionnelle, laquelle conserve pour les stoïciens une forme de légitimité dans le cadre de la petite cité :

Ici l'on répond que tout sans doute est aux dieux, mais que tout ne leur est pas dédié ; et que, si nous sommes en présence d'un acte sacrilège, c'est à propos d'objets assignés par la religion à la divinité (quae religio numini adscripsit) [...] donc comme on juge sacrilège celui qui vole un objet sacré, lors même que chaque fois le lieu où est passé le fruit du larcin est compris dans les limites de l'univers (intra terminos mundi), ainsi, même au sage on peut faire un larcin ; ce sera prendre en effet non sur son universel domaine, mais sur le domaine qui est enregistré à son nom. (VII, 7, 3-4, trad. F. Préchac 1927)

Sénèque ainsi établit une analogie entre la propriété universelle des dieux et celle des sages : alors que dans l'absolu, c'est-à-dire selon la nature, la totalité de l'univers appartient aux dieux, on peut néanmoins commettre un sacrilège en sortant les objets consacrés à la divinité de l'espace qui leur est assigné. La religio et son partage entre le sacré et le profane n'est pas abolie, malgré l'homogénéité de l'espace naturel appartenant dans son ensemble à la divinité. De façon semblable, Sénèque précise qu'il est possible de voler le sage, or cette remarque ne peut prendre sens que dans le cadre de la cité cosmique où « tout appartient aux sages », thèse que nous pouvons faire remonter à la conclusion du syllogisme de Diogène, que Sénèque suppose connue de ses lecteurs et, sans doute, de Bion lui-même.

Quant à la deuxième partie de son raisonnement, elle conduit à justifier le vol dans les temples (qui était un acte d'impiété passible de poursuites ${ }^{32}$ ) : on ne peut rien soustraire à la propriété divine car il n'est aucune partie de l'espace qui ne lui appartienne. La filiation de l'argument là aussi est cynique, puisque Diogène nous est traditionnellement présenté comme mangeant dans les temples ${ }^{33}$; or chez Bion cette autorisation n'est pas réservée aux sages, mais étendue à tous.

Ces deux arguments d'inspiration cynique sont cependant articulés dans une structure pro et contra, dont la forme entre plutôt en résonnance avec

31. DL VI, 72 (= SSR V B 353), raisonnement qui pouvait aussi bien servir de justification à la mendicité ( $c f$. Cratès, Lettres, $27=S S R$ VH 114 ; nous trouvons également le même syllogisme attribué à Socrate chez Diogène, Lettres, 10, $1=$ SSR V B 540), que d'autorisation pour le cynique de consommer les offrandes aux dieux.

32. Cf. Thür 2006.

33. DL VI, $73=$ SSR V B 353. Cependant il ne s'agit pas réellement d'un vol car il était d'usage dans l'Antiquité de permettre aux pauvres de manger les offrandes en nourriture aux dieux et les restes des sacrifices, $c f$. Veyne 2000 p. 14. 
l'Académie sceptique et, en particulier, avec Carnéade ${ }^{34}$. Ce genre d'arguments témoigne de la formation dialectique dont Bion avait bénéficié, qui lui faisait dire que tout argument pouvait être renversé ${ }^{35}$. Il permet aussi de mieux comprendre pourquoi étrangement Diogène Laërce a pu situer Bion, dans son quatrième livre consacré à l'Académie, entre Arcésilas et Carnéade ${ }^{36}$. Le seul motif n'était peut-être pas simplement son identification comme élève de Cratès d'Athènes (qui prendra la tête de l'école après que Bion l'eût quittée), mais son mode d'enseignement pouvant, par certains aspects, s'inscrire dans une filiation académicienne ${ }^{37}$. Cependant, il ne s'agissait pas pour lui de mener à la suspension du jugement ${ }^{38}$ concernant le fait de savoir s'il est permis ou non de prendre ce qui appartient aux dieux, mais de montrer à quel point les impératifs pratiques fondamentaux de la piété (la séparation entre sacré et profane) entrent en contradiction avec la déclaration pieuse tout aussi fondamentale d'après laquelle « tout appartient aux dieux ». La religio est ainsi déclarée irrationnelle parce que contradictoire. Mais si la piété traditionnelle est condamnée, ce développement dialectique n'implique nullement que les dieux n'existent pas. Bien au contraire, il semble vouloir corriger les représentations communes par une théologie rigoureuse. Il n'en va pas de même de sa contestation des rites funéraires.

Bion, en effet, partageait avec les cyniques ${ }^{39}$ le mépris des rites mortuaires dont le respect était si crucial chez les Anciens et dénonçait leur absurdité en mettant en valeur la contradiction des croyances qui les fondaient :

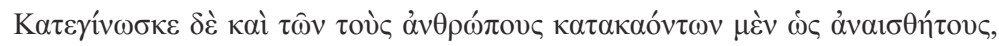

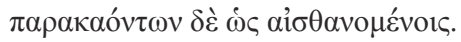

Il [scil. Bion] blâmait aussi ceux qui brûlent les cadavres en les tenant pour insensibles et déposent à côté d'eux des lampes allumées en les tenant pour sensibles ${ }^{40}$.

34. Dont l'argumentation pro et contra sur la justice fit sensation à Rome (Lactance, Institutions divines, $\mathrm{V}, 15)$.

35. Plutarque, De Pythiae oraculis, 5, 396E (= F10 Kindstrand 1976). 28-66).

36. Plus précisément, l'ordre de succession est Arcésilas, Bion, Lacydès, Carnéade (DL IV,

37. La question de savoir pourquoi Diogène Laërce a placé Bion dans l'Académie a laissé les commentateurs perplexes, dans la mesure où ils n'ont décelé nulle trace d'influence de l'enseignement de l'Académie dans sa doctrine, la réponse la plus raisonnable paraissant être son statut d'élève de Cratès d'Athènes (DL IV, 51). Cf. Kindstrand 1976 p. 19, 57-58 qui nuance cette vue en étudiant l'influence possible de l'enseignement éthique de Polémon.

38. Bien que Numénius rapporte une anecdote, qu'il estime lui-même douteuse, d'après laquelle Arcésilas aurait adopté la suspension du jugement par peur des attaques de Bion (Diocles ap. Numenium cité par Eusèbe, PE, XIV 6, 6 = T23 Kindstrand 1976). Pour les traits sceptiques de Bion, $c f$. Kindstrand 1976 p. 77-78.

39. Cf. S. Husson 2011 p. 134-138.

40. DL IV, 48 (= F71 Kindstrand 1976), trad. T. Dorandi. On peut remarquer l'effet

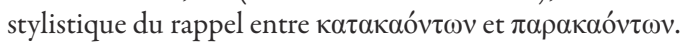


Cependant, entre ces deux conceptions contradictoires de la mort, Bion avait tranché en faveur de l'insensibilité des cadavres.

Il [scil. Bion] avait coutume de dire que le chemin vers l'Hadès est facile : on s'y rend en tout cas les yeux fermés.

Bion disait qu'il y a deux choses qui enseignent la mort : le temps avant la naissance et le sommeil ${ }^{41}$.

La désacralisation de la mort s'appuie ainsi sur la thèse de la disparition de la personnalité consciente. Les fragments ne nous permettent pas de savoir quelle conception physique avait pu conduire Bion à cette conclusion. Il n'en reste pas moins que nier l'existence d'une « vie après la mort » conduit à refuser toute la mythologie relative à l'au-delà et aux enfers, que Bion critiquait par ailleurs puisqu'il estimait qu'il aurait été plus pénible pour ceux qui sont punis dans l'Hadès de porter des récipients pleins plutôt que des récipients percés ${ }^{42}$. Mais la réflexion de Bion allait sans doute beaucoup plus loin, puisque nous savons qu'il passait les récits sur les dieux au crible de la rationalité morale.

Nous savons, par exemple, que Bion ironisait sur Zeus punissant les enfants des coupables, comme si les médecins ne soignaient pas leur patient mais des membres de la famille ${ }^{43}$. Il s'agit là d'une remise en cause des cycles mythiques les plus traditionnels - relevant d'une conception archaïque de la faute objective, comprise comme souillure transmissible à la descendance - à la lumière de la conception largement acquise au $\mathrm{IV}^{\mathrm{e}} \mathrm{s}$. av. J.-C. de la responsabilité subjective de l'individu ${ }^{44}$.

Il estimait également qu'il était ridicule de demander à Zeus une bonne descendance, alors qu'il n'était pas parvenu à se la procurer pour lui-même ${ }^{45}$ - remarque qui dénonçait, une fois de plus, le manque de clairvoyance des dieux mythologiques.

Bion jugeait donc les pratiques religieuses ${ }^{46}$ ainsi que les récits mythiques irrationnels et se moquait des dieux du panthéon dans un style cynique : cela suffisait en soi pour faire de lui un atheos, un impie, aux yeux du public antique, et ceci aussi bien au niveau pratique qu'au niveau de la négation théorique des dieux, en tant qu'ils sont objets d'une croyance commune ; mais pouvons-nous le considérer comme un athée au niveau philosophique?

41. DL IV, 49 (= F66 Kindstrand 1976), trad. T. Dorandi et Gnomologium Vaticanum 160 (= F67 Kindstrand 1976).

42. DL IV, 50 (= F28 Kindstrand 1976).

43. Plutarque, De sera numinis vindicta, 19, 561C (= F27 Kindstrand 1976).

44. Cf. Gernet 1917 p. 342-348.

45. Clément d'Alexandrie, Protreptique, IV 56, 1 (= F29 Kindstrand 1976).

46. Nous trouvons également chez lui ce qui semble une dénonciation de la superstition (Plutarque, De la superstition, 7, 168D, F30 Kindstrand), ainsi qu'une critique des devins (D. L. II 135 = F32 Kindstrand 1976), mais dans des fragments trop allusifs pour pouvoir nous éclairer. 
Les philosophes qui, depuis Xénophane, se sont attaqués aux pratiques et aux représentations religieuses leur substituaient, pour la plupart, une conception rationnelle de la divinité en désaccord avec elles. Pouvons-nous donc considérer que Bion niait absolument l'existence des dieux, comme les athées du livre X des Lois de Platon qui en font une construction humaine (889e), ou qu'il visait simplement à en produire une conception épurée ? Un témoignage montrant que Bion prenait part aux débats théologiques de son temps pourra peut-être nous mettre sur la voie.

\section{Contre les dieux des philosophes}

Bion est, en effet, cité dans deux passages du traité de l'épicurien Démétrius Lacon, De la forme du dieu ${ }^{47}$ (PHerc. 1055). Dans la colonne XVIII du papyrus, Démétrius expose un argument visant à réfuter l'anthropomorphisme des dieux des épicuriens : si le dieu a forme humaine, il aura aussi des yeux et de la sorte pourra souffrir d'ophtalmie et des inférences similaires pourront aussi être faites à propos des autres sens. Le texte lisible de la colonne s'arrête là, mais le sens de l'argumentation est clair : il est évident que des dieux aux sensations douloureuses entreraient en contradiction avec le parfait bonheur qui, pour Épicure, définit les dieux, d'après la prolepsis que nous en avons ${ }^{48}$. Démétrius continue en introduisant un autre argument :

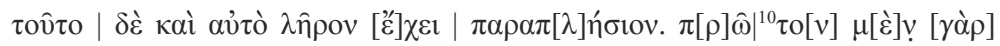

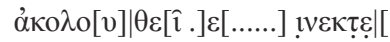

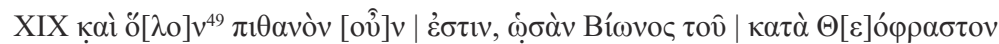

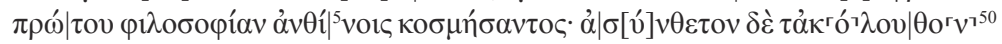

47. Sur la question du titre de l'œuvre dont le papyrus PHerc. 1055 présente des fragments, voir la discussion de Santoro 2000 p. 23-28.

48. Cf. Épicure, Lettre à Ménécée, 123-124, Maximes capitales, I ; Cicéron, De la nature des dieux, I 43-51.

49. ö[ $[\lambda o] v$ : reconstruction de M. Santoro ; de Falco 1923 lit oỏ $[\delta \bar{\varepsilon}] v$ et D. Delattre et T. Dorandi (dans Delattre \& Pigeaud 2010) traduisent dans ce sens : « et l'argument n'emporte aucunement la conviction assurément $\gg$. Pour notre part, il nous semble que le rappel de la formule d'après laquelle Bion, à cause de son style brillant et bigarré fut « le premier à avoir paré la philosophie de fleurs » (allusion aux manteaux colorés des prostituées athéniennes, voir aussi T12 Kindstrand 1976 ; sur le style de Bion et l'interprétation du terme ỏv0ívorc, voir Kindstrand 1976 p. 49-55, surtout p. 51-52 et Santoro 2000 p. 157-158) est visiblement ironique : c'est un reproche déguisé en éloge. Bion, à cause de la séduction exercée par son écriture aurait été le premier à avoir prostitué la philosophie. Mais si son écriture est séduisante, elle doit, en principe, emporter la conviction, dont la suite montrera qu'elle n'est qu'en partie fondée. Gigante et Indelli 1978, qui lisent o[...]v choisissent de ne pas proposer de restitution.

50. Ce passage pose une difficulté textuelle. En effet, le texte du papyrus -

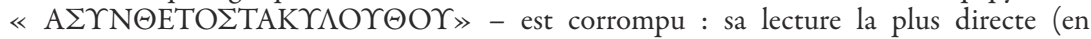

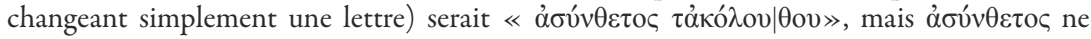
gouverne pas de complément au génitif. C'est pourquoi M. Santoro, à la suite de Philippson, corrige le génitif en optant pour le nominatif $\tau \dot{\alpha} \kappa o ́ \lambda o v \theta o v$. M. Gigante, quant à lui, opte pour 


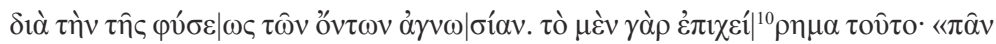

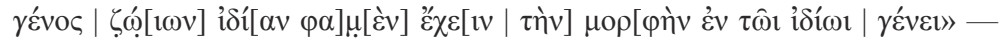

Mais cet argument même comprend une incongruité presque équivalente. D'abord, il s'ensuit... que [...]

XIX ... et [l'argument] est, donc, tout à fait persuasif, comme si Bion avait été le premier (à ce que dit Théophraste) à parer la philosophie de fleurs. Ce qui en découle, cependant, est incohérent, à cause de l'ignorance de la nature des êtres. Cet épichérème, en effet : « nous disons que chaque genre de vivants a une forme particulière dans son genre particulier ${ }^{51}[\ldots]$

De l'argument annoncé, nous savons sans ambiguïté qu'il est attribuable à Bion, connu pour son style théâtral et humoristique. Ainsi, Démétrius rapporte le jugement de son maître Théophraste qui reprochait à Bion d'avoir apporté trop d'ornements au discours philosophique ${ }^{52}$. Nous savons d'autre part qu'il contenait la formule « nous disons que chaque genre de vivants a une forme particulière dans son genre particulier », qualifiée d'épichérème.

L'épichérème est défini, dans ce qui apparemment est une glose présente dans le livre VIII des Topiques d'Aristote, comme un syllogisme dialectique ${ }^{53}$. Nous savons par ailleurs que Théophraste avait écrit un ouvrage de dix-huit livres consacré à ce genre d'arguments ${ }^{54}$. Chez Aristote l'expression

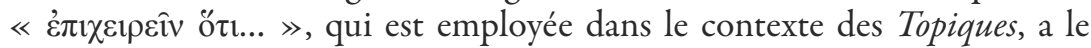
sens d'entreprendre une démonstration pour réfuter l'adversaire ${ }^{55}$ et le

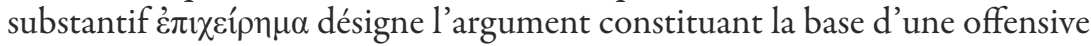

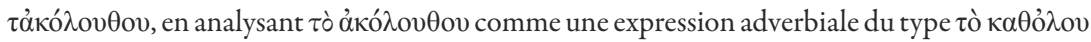
(Santoro 2000 p. 159-161) ; néanmoins une rapide recherche sur le TLG montre qu'alors que

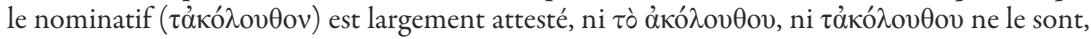
ce qui constitue un argument de poids en faveur du nominatif.

51. Démétrius Lacon, De la forme du dieu, XVIII 7-XIX 13 (= T13, F26A Kindstrand 1976), éd. Santoro 2000.

52. « Il était théâtral et il excellait à ridiculiser (toutes choses), utilisant alors des expressions vulgaires pour désigner les choses. Du fait qu' il mélangeait tous les styles, on dit qu'Ératosthène dit [à son propos] que Bion fut le premier à revêtir la philosophie d'un manteau de fleurs » (DL IV, 52 = T12 Kindstrand 1976, trad. T. Dorandi, très légèrement modifiée). Kindstrand (1976 p. 39-49) décèle dans son style une influence à la fois du gorgianisme et du spoudaiogeloion cynique. Pour l'attribution de la paternité de la formule à Théophraste et non à Ératosthène, Kindstrand 1976 p. 53-55, Santoro 2000 p. 157-158, voir aussi supra n. 49.

53. Aristote, Topiques, VIII, 11, 162a15-18 : «Un philosophème est un syllogisme démonstratif, un épichérème un syllogisme dialectique, un sophisme un syllogisme éristique, un aporème le syllogisme dialectique d'une contradiction $\gg$. Ce passage, dans la mesure où il n'est pas commenté par Alexandre, est très certainement une glose ( $c f$. Brunschwig 2007 n. ad. loc.).

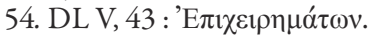

55. Top. 128 b 26, 31, $32 ; 150$ a 15; 163a 2, comme le signale Bonitz 1870 p. 283 1. 1-2. On peut voir dans cet usage offensif un héritage de l'usage de ce verbe dans des contextes militaires (cf. LSJ s.v., 2). 
dialectique :

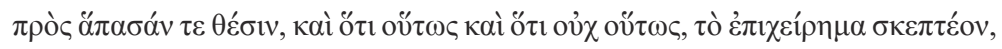

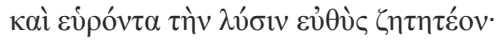

À propos de toute thèse, qu'elle énonce qu'il en est ainsi ou qu'il n'en est pas ainsi, il faut examiner le moyen de l'attaquer ( $\tau$ ò $̇ ં \pi \chi \varepsilon i ́ p \eta \mu \alpha)$, et dès qu'on l'a trouvé, il faut en chercher immédiatement la solution ${ }^{56}$.

Nous pouvons donc considérer que l'épichérème est dans ce contexte un argument qui devait sans doute prendre la forme d'un syllogisme adapté au contexte dialectique, c'est-à-dire dont les prémisses, de l'ordre des endoxa, doivent être explicitement accordées par l'interlocuteur visé, et qui a pour fonction de réfuter la thèse (c'est-à-dire la réponse au problème) adoptée par lui. Alors qu'il n'est envisagé par Aristote que dans le cadre d'un entraînement intellectuel, Bion en faisait visiblement usage dans la polémique philosophique. Dans une optique dialectique, il cherchait sans doute à montrer que les épicuriens ne pouvaient pas admettre en même temps l'anthropomorphisme des dieux et l'évidence sensible qui montre que chaque espèce d'êtres vivants se distingue par une configuration physique particulière. De plus, l'exposé de Démétrius montre que l'épichérème ne commençait pas par la proposition à réfuter (les dieux ont forme humaine) mais par une thèse que les épicuriens sont censés admettre et qui permettra de la réfuter. La structure argumentative est donc la suivante :

Majeure : Vous admettez ${ }^{57}$ que chaque genre de vivants a une forme particulière dans son genre particulier ;

Mineure : Or vous admettez aussi que les dieux et les hommes appartiennent à deux genres différents ;

Conclusion : Donc vous admettez que les dieux ne peuvent pas avoir la même forme que les hommes.

Bion se contentait-il de présenter cette structure ? Rien n'est moins sûr puisque Démétrius signale que l'argument est persuasif ( $\pi(\theta \alpha v o ́ v)$, mais qu'une partie de sa force de persuasion vient du style fleuri de Bion. Il est donc probable que celui qu'on a aussi qualifié de sophiste ${ }^{58}$ ne se contentait pas d'un simple schéma mais avançait chaque étape en la justifiant de multiples manières, ce qui peut faire de lui un vecteur de transition entre l'origine dialectique de l'épichérème et le sens qu'il prendra dans la tradition rhéto-

56. Top., 163a 36-b 1, trad. J. Brunschwig 2007.

57. Ou « vous dites »: nous supposons ici que l'épichérème était formulé à la deuxième personne et que Démétrius le reprend à la première personne : « nous disons que chaque genre de vivants etc. », mais on ne peut pas du tout exclure qu'il ait été formulé directement à la première personne. En tout cas, il faut considérer que $\varphi \alpha \mu \varepsilon^{\prime}$ en XIX, 11 n’est pas une incise, mais fait partie de l'argument, et signale son caractère dialectique (pour une interprétation contraire, voir la traduction Dorandi \& Delattre dans Delattre \& Pigeaud 2010 ad. loc.).

58. Plutarque, Contre Colotès, 1126 A (= T28 Kindstrand 1976). 
rique $^{59}$. Sans pouvoir restituer son style nous pouvons néanmoins envisager certains de ses arguments.

Tout d'abord, la majeure, enjeu central du débat, n'était pas sans pertinence, puisque dans la colonne XXIII, comme nous le verrons, Démétrius explicite à quelles conditions un épicurien peut l'admettre, ce qui montre

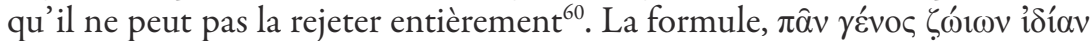

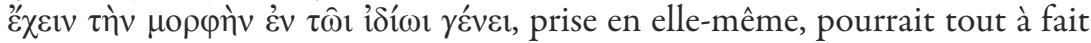
être comprise dans un sens aristotélicien : comme le montre Aristote dans le traité des Parties des animaux, chaque genre ${ }^{61}$ de vivants possède une configuration physique qui lui est propre, déterminée par son eidos et les fonctions qui lui sont liées ; mais un épicurien se doit de l'admettre au niveau des réalités accessibles à la sensation. C'est, en effet, une proposition constamment confirmée par cette dernière ; bien plus l'explication épicurienne de la formation des notions générales en nous, à savoir les prolepseis, l'implique. Celles-ci se forment par répétition de l'expérience de sensations semblables, jusqu'à la constitution d'un schéma mental qui permet de structurer et d'anticiper les données sensibles ${ }^{62}$. Or si les prolepses se distinguent entre elles et nous permettent de distinguer les genres, c'est parce qu'elles se sont formées à partir de l'afflux de simulacres ayant eux-mêmes des atomes et des structures différentes, autrement dit émanant de vivants ayant des configurations différentes. Un épicurien ne peut donc pas refuser le fait que les genres d'êtres vivants

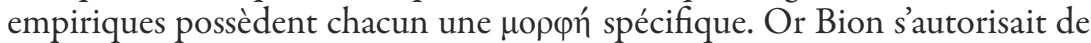
cet accord pour étendre cette proposition à tous les genres d'êtres vivants, même ceux dont le statut est plus problématique, à savoir les dieux. Nous ne pouvons pas savoir s'il justifiait cette extension, mais il aurait pu s'autoriser du principe épicurien d'isonomie, principe fondamental qui postule a priori une

59. Dans la tradition rhétorique, en effet, l'épichérème est devenu une version amplifiée de l'enthymème (le syllogisme rhétorique) dont on multiplie à loisir les étapes - voir par exemple Rhétorique à Hérennius, 2, 28. Nous pouvons aussi trouver chez Cicéron une présentation à la fois rhétorique et syllogistique de l'épichérème, qui montre la persistance, au-delà du contexte rhétorique, de la référence à la tradition dialectique (péripatéticienne en particulier) concernant l'épichérème : Cicéron, De l'invention, I, 57-77, cité et analysé par Braet 2004.

60. Nous voyons ainsi que Bion est considéré, malgré son originalité stylistique, comme un adversaire sérieux soulevant des objections pertinentes. Comme l'ont montré M. Gigante et G. Indelli 1978 l'attitude des épicuriens à l'égard de Bion, que jusqu’à J. F. Kindstrand on considérait comme uniquement polémique, implique qu'ils le considéraient au contraire comme un interlocuteur légitime.

61. Ici nous pouvons considérer que $\gamma \varepsilon ́ v o \varsigma$ est utilisé sans impliquer de distinction entre genre et espèce.

62. DL X, 32-33, cf. Long \& Sedley 1987 p. 88-89. Remarquons que cette polémique entre Bion et Démétrius suppose que les dieux des épicuriens ont une existence objective et ne sont pas des constructions mentales, contrairement à l'interprétation que Long \& Sedley 1987 p. 144-147 donnent des dieux d'Épicure. Pour une interprétation contraire, cf. Mansfeld 1993. 
homogénéité du réel et de ses lois et qui permet, certes à certaines conditions, d'étendre notre connaissance au-delà des évidences sensibles immédiates ${ }^{63}$.

L'objection de Bion n'est donc pas, pour un épicurien, dénuée de fondement ; c'est pourquoi Démétrius entreprend, en trois temps, d'y répondre. Il procède tout d'abord à une réfutation par la conséquence : $\alpha \hat{\alpha} \mid \sigma\left[{ }^{\prime}\right] v \theta \varepsilon \tau$ ov

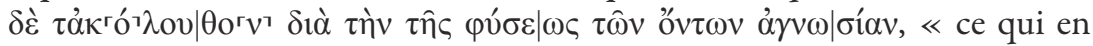
découle, cependant, est incohérent, à cause de l'ignorance de la nature des êtres $\gg$ (XIX, 5-9).

La colonne XX montre que Démétrius commence par démontrer que la majeure de l'épichérème ne permet pas seulement de réfuter la théologie épicurienne, mais certaines théologies philosophiques concurrentes, à savoir les théologies cosmiques et astrales :

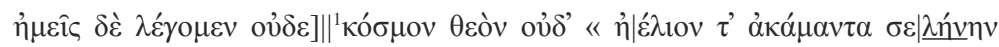

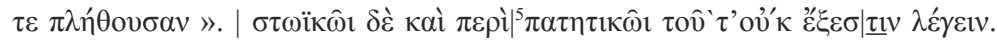

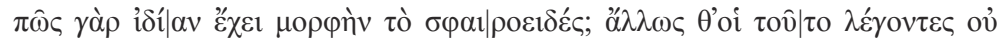

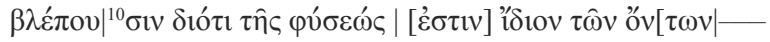

[Nous ne disons pas que] le monde est un dieu ni « le soleil inépuisable ni la lune pleine ${ }^{64}$. Or à un stoïcien ou à un péripatéticien il est impossible de le dire. En effet, comment ce qui est de forme sphérique possède-t-il une forme particulière ? En outre, ceux qui disent cela ne considèrent pas pourquoi ce qui est particulier à la nature des êtres [est] ${ }^{65} \ldots$

L'argument est purement dialectique et vise à établir que les épicuriens sont en fait plus cohérents que leurs adversaires. Les étapes nous semblent devoir être les suivantes :

1- Nous (épicuriens) disons que chaque genre de vivants a une forme particulière dans son genre particulier;

2 - Une conséquence en est que tout genre d'êtres qui n'a pas de forme qui lui soit particulière n'est pas un être vivant ;

3 - Ce monde ainsi que les astres sont de forme sphérique ;

4 - Donc la forme sphérique est commune à plusieurs genres d'êtres;

5 - Donc (en vertu de 2) ce monde, ainsi que les astres ne sont pas des êtres vivants ;

6 - Les dieux sont des êtres vivants ;

7 - Donc (en vertu de 5 et 6) ce monde et les astres ne sont pas des dieux, ce qu'effectivement nous disons.

L'admission de 1, 3 et 6 entraîne celle de la conclusion 7. Donc toute école prête à admettre ces propositions devrait, comme les épicuriens, en

63. Cf. Mugler 1956.

64. Homère, Il. XVIII 484.

65. Démétrius Lacon, De la forme du dieu, XX, éd. Santoro 2000. 
conclure que le monde et les astres ne sont pas des dieux, ce que, comme le signale Démétrius, un stoïcien et un péripatéticien ne peuvent pas faire.

L'attaque ne peut porter que si, en plus de la première proposition, les écoles concurrentes sont obligées d'admettre la troisième et la sixième. Comme nous l'avons vu, la première proposition ne pose aucun problème dans un cadre aristotélicien et sans doute est-il aussi possible de lui donner un sens dans un cadre stoïcien, dans la mesure où les stoïciens, comme les épicuriens, admettent la formation de notions génériques à partir de l'expérience sensible ${ }^{66}$. Ces deux écoles partagent aussi le fonds commun hérité, entre autres, de la physique platonicienne qui attribue au monde et, bien entendu, aux astres, une forme sphérique ${ }^{67}$. Quant à l'idée qu'un dieu est un être vivant (proposition 6), celle-ci fait partie du fonds commun de toute théologie rationnelle $e^{68}$. À partir de là, si nous considérons que le monde et les astres appartiennent nécessairement à deux genres d'êtres différents puisque les uns sont une partie de l'autre, les partisans des théologies cosmiques et astrales seraient, dans un contexte dialectique, obligés de se dédire.

Sans pouvoir assurer que Démétrius exposait toutes les étapes de sa réfutation par la conséquence, nous pouvons néanmoins avancer que celle-ci démontrait que la majeure de l'épichérème de Bion constituait un véritable problème, non seulement pour la théologie épicurienne, mais pour celles des écoles concurrentes, ainsi que pour Bion lui-même, en tant qu'élève de Théophraste $^{69}$. Un défi que chacune avait à relever. C'est pourquoi Démétrius entreprend de le faire pour sa propre école.

En plus de présenter une conséquence rendant la théorie des adversaires incohérente, Démétrius annonçait que l'objection de Bion présentait « une incongruité ( $\lambda \hat{n} \rho \circ v)$ presque équivalente » (XVIII 7-9) à l'objection précédente, c'est-à-dire celle qui inférait du fait que les dieux des épicuriens possèdent, comme les hommes, des organes sensoriels, le fait qu' ils en possèderont également les infirmités (col. XVIII), et ne jouiront donc pas d'un bonheur parfait. Or celle-ci est incongrue parce que, comme l'objection suivante, elle poursuit indûment l'analogie entre les êtres vivants sensibles et les dieux. Pour un épicurien, certes, on peut inférer une homologie de forme entre corps humain et divin, mais celle-ci ne peut être poursuivie que dans une certaine mesure, c'est-à-dire lorsque rien d'autre dans la prolepsis du dieu

66. Cf. Aëtius, 4, 11, 1-4 ; Cicéron, Académiques, II 21, Long \& Sedley 1987 p. 241.

67. Cf. Platon, Timée, 33b, 38c-d ; Aristote, Du ciel, II, 286b10-287b21 ; pour les stoïciens, par exemple, Stobée I 166, 4-22 (= SVF I ,99).

68. Cf. par exemple, pour Aristote, Bodéüs 1992 p. 51.

69. Pour l'attribution à Théophraste d'une théologie cosmique et astrale, $c f$. Cicéron, $\mathrm{La}$ nature des dieux, I, 13, 35. On connaît par ailleurs les similitudes entre l'exposé de la théologie épicurienne dans le premier livre de l'ouvrage de Cicéron et celui de Démétrius dans De la forme $d u$ dieu, qui peuvent indiquer au moins une source commune, cf. Santoro 2000 p. 43-50. 
ne s'y oppose, et, en particulier, sa béatitude ${ }^{70}$. Cette objection procède, en fin de compte, comme la suivante, d'une « ignorance de la nature des êtres » (XIX 7-9), en particulier d'une ignorance du fait que lorsqu'il s'agit de la divinité, c'est de la prolepsis qu'il faut partir et c'est elle qui sera la pierre de touche de tout raisonnement analogique.

Elle ignore d'autre part que les dieux, bien qu'ils nous soient connus, ne le sont pas par la sensation, de sorte que nous ne pouvons pas leur appliquer toutes les propriétés que la sensation découvre aux vivants. Démétrius poursuit ainsi en explicitant le processus particulier par lequel les simulacres des dieux nous parviennent : leurs atomes plus ténus ne peuvent pas provoquer de sensations mais parviennent néanmoins à l'âme pour former la prolepsis correspondante (col. XXI) ; c'est également la ténuité des corps divins qui permet d'expliquer leur immortalité a contrario des corps accessibles à la sensation, tous corruptibles (col. XXII) ${ }^{71}$.

Après avoir expliqué en quoi la nature des êtres divins, accessibles essentiellement par la raison, differe de celle des êtres accessibles par la sensation, Démétrius peut conclure :

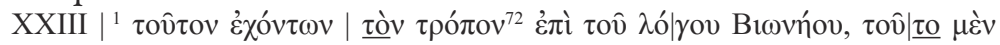

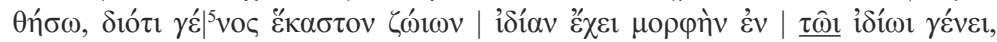

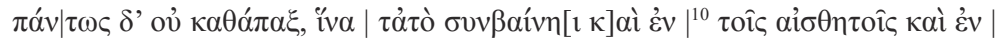

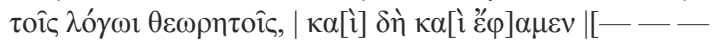

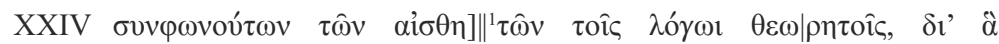

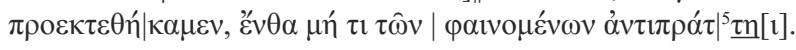

XXIII [...] qui ont cette tournure concernant l'argument de Bion, voici ce que je poserai, que « chaque genre de vivants a une forme particulière dans son genre particulier », mais en aucun cas d'une manière absolue, afin que la même chose s'ensuive à la fois dans les réalités sensibles et dans celles qu'on peut considérer par la raison, et qui plus est nous disions [...]

XXIV les réalités sensibles s'accordant avec celles qui sont considérées par la raison, en vertu des raisons que nous avons précédemment exposées, lorsque rien parmi les phénomènes ne s'y oppose ${ }^{73}$.

Autrement dit, en tant qu'épicurien, j'accepte la majeure de Bion, à savoir que « chaque genre de vivants a une forme particulière dans son genre

70. La béatitude, en effet, n'est possible que pour un être rationnel. Or la raison ne se rencontre que chez les animaux qui ont forme humaine, comme le dit Cicéron (De la nature des dieux, I, 48). Cependant, si la forme humaine des dieux peut être découverte par ce raisonnement, elle est néanmoins immédiatement donnée à l'imagination des hommes, lorsque leur âme en reçoit directement des simulacres, soit à l'état de veille, soit en rêve, comme l'unanimité des peuples à révérer des dieux anthropomorphes en témoigne (I, 46). Sur ce double mode de connaissance des dieux, $c f$. Mansfeld 1993 p. 209-210.

71. Ce qui correspond à Cicéron, I, 44-45 et 49.

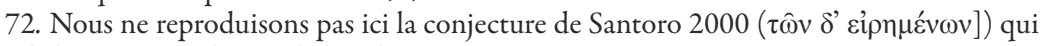
précède les premières lettres de la colonne.

73. Col. XXIII 1-XXIV 6 (= F26B Kindstrand 1976, part.). 
particulier », mais en la restreignant aux vivants observés par la sensation et en refusant de l'étendre à ceux qui sont observés par la raison, à savoir les $\operatorname{dieux}^{74}$. De la sorte on pourra, par un raisonnement analogique, attribuer la même caractéristique à la fois aux hommes et aux dieux, à savoir que, de la présence commune de la raison en eux, leur anthropomorphisme s'ensuivra.

Cependant, Démétrius prend soin de préciser que cette restriction n'est en aucun cas un principe de méthode ; bien au contraire, la possibilité de généraliser les observations nées de l'expérience et d'étendre les vérités connues par la sensation aux réalités non visibles est l'outil le plus puissant de l'épistémologie épicurienne. Le traité de Philodème Sur les signes témoigne à la fois des attaques que les épicuriens avaient à subir de leurs adversaires et de leurs ripostes. Or, Philodème rapporte que Démétrius avait participé activement à la polémique : il avait, en particulier, écrit un ouvrage (Philodème, De signis, XXVIII 45 sq.) où étaient exposés les arguments des adversaires contre les modes d'inférence épicuriens, ainsi que les réponses qu'il leur donnait.

(1) Mais [ces erreurs, Démétrios] les a reprises en détail dans sa discussion dialectique ( $\delta 1 \alpha \lambda \varepsilon \gamma o ́ \mu \varepsilon v o \varsigma)$.

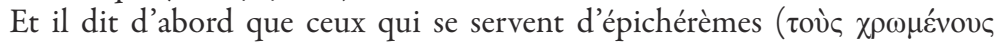

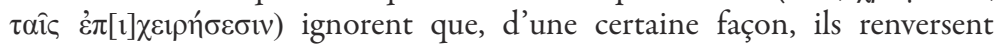
les arguments contre eux-mêmes sans pudeur. En effet, les arguments qu'ils combinent quand ils rejettent le mode selon la similitude ( $\tau$ òv $\kappa \alpha \tau \grave{\alpha}$

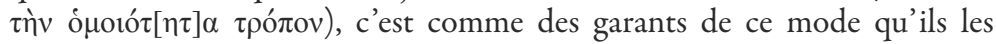
présentent ! (2) En tout cas, chaque fois qu'ils rencontrent la question de l'origine d'une inférence comme : « si les vivants de notre expérience ( $\tau \grave{\alpha}$

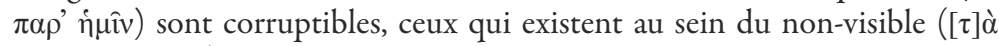

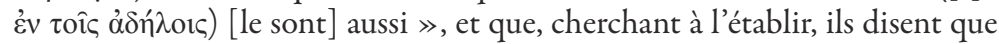
quelques-uns d'entre eux, tout en étant semblables par le genre, présentent entre eux une différence qui est liée aux airs [30], aux nourritures et à toutes les autres variables qu'on voudra, ils partent des phénomènes dont nous avons

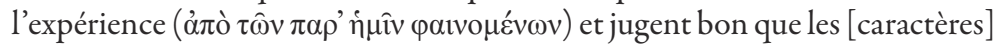
fort semblables vaillent pour ces [vivants non visibles] : voilà comme ils procèdent ! (3) Et chaque fois que l'existence, dans notre expérience, d'êtres

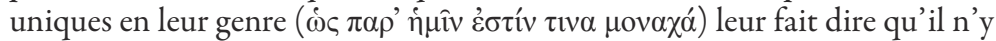

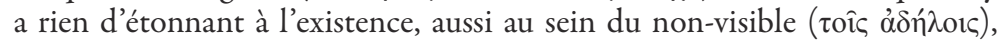
de quelque nature différant des [vivants] que nous avons rencontrés, c'est

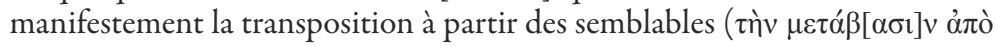

74. Les dieux des épicuriens, en effet, sont, à ce qu'il parait, les seuls êtres vivants accessibles par la raison, c'est-à-dire dont la connaissance n'est pas due à la sensation mais, soit à des images perçues dans les rêves, soit à la prolepsis (cf. supra n. 68). Les seuls autres êtres connus uniquement par la raison sont les principes des corps, à savoir les atomes et le vide, ainsi que les composés infra-phénoménaux. Or il ne semble pas, à notre connaissance, que les épicuriens aient conçu que certains de ces êtres eussent pu être des êtres vivants et qu' ils aient, donc, pensé l'existence d'une vie microbiologique, bien que cette possibilité ne soit pas, en soi, contradictoire avec leur physique. 
$\tau \hat{\omega} v$ ónoí $\omega v)$ qu'ils pratiquent; et il en va d'une manière fort semblable pour tout le reste. Conséquence : [leur raisonnement] implique son renversement radical $^{75}$.

Ici Démétrius s'en prend à ceux qui « rejettent le mode selon la similitude », c'est-à-dire les adversaires, essentiellement stoïciens, qui refusent qu'on puisse inférer des vérités sur le non-visible à partir d'une ressemblance avec ce dont nous avons l'expérience ( $\tau \grave{\alpha} \pi \alpha \rho$ ' $\dot{\eta} \mu \hat{\imath} v)$. Il montre ainsi comment il est impossible de réfuter ce mode d'inférence sans recourir à lui. Par exemple (section 3), si l'on objecte aux épicuriens qu'il est impossible de savoir si ce qui fait partie du non-visible est semblable à ce dont nous avons l'expérience, parce que cette expérience nous montre qu'il existe des objets uniques en leur genre, ces objecteurs présupposent bien une ressemblance entre le visible et le non-visible dans leur argumentation. De même (section 2), s'ils contestent l'incorruptibilité des dieux des épicuriens au motif que les réalités dont nous avons l'expérience sont toutes corruptibles, ils opèrent également une transposition du visible au non-visible à partir du mode de la ressemblance. En l'occurrence la défense de Démétrius n'est peut-être pas la plus pertinente : les adversaires s'exprimant dans un cadre clairement dialectique (section 1), il est normal qu'il recourent aux principes admis par ceux qu'ils entreprennent de réfuter « en utilisant des épichérèmes ».

Face à Bion, la situation dialectique était, en un certain sens, inversée. Bion s'appuyait explicitement sur « le mode selon la similitude » pour l'étendre au non-visible et en conclure que les épicuriens devraient soutenir que les dieux ont une forme qui leur est strictement particulière. Le reproche d'utilisation inconsciente de ce mode ne pouvait pas lui être fait. Il en prônait au contraire un usage incontrôlé. La réponse de l' épicurien, de ce fait, était beaucoup plus pertinente : «le mode selon la similitude »s'applique, mais non pas absolument, c'est-à-dire tant que « rien parmi les phénomènes ne s'y oppose », et aux phénomènes nous pourrions ajouter la prolepsis des dieux.

Cependant, malgré ces polémiques, nous ne pouvons pas savoir en toute certitude si Bion, tout en rejetant les dieux traditionnels ainsi que les dieux des épicuriens, n'admettait pas d'autres dieux (ou un autre dieu) de philosophes (quoique nous puissions en douter), mais nous pouvons comprendre qu'il existait, pour ses contemporains, au moins trois raisons de penser qu'il était athée : l'impiété de son comportement pendant la majeure partie de sa vie ; son rejet des dieux de la cité, ainsi qu'enfin sa critique de théologies philosophiques alternatives.

75. Philodème, De signis, XXIX, 24-XXX, 15, trad. Boulogne et al. (dans Delattre \& Pigeaud 2010 p. 555-556), très légèrement modifiée. Les citations du texte entre parenthèses sont issues de l'édition De Lacy (De Lacy \& De Lacy 1941), dans la mesure où il apparaissait que dans ces passages, ce texte avait été validé par les traducteurs. Les subdivisions entre parenthèses ont été ajoutées par nous. 


\section{BIBLIOGRAPHIE}

BoDÉÜs, R. 1992 : Aristote et la théologie des vivants immortels, Québec/Paris, 1992 (Noêsis).

Bonitz, H. 1870 : « Index Aristotelicus » dans I. Bekker (dir.) Aristotelis opera, vol. V, Berlin, 1870.

Boulogne, J. et al. 2010 (trad.) : Philodème de Gadara, Les [phénomènes] et les inférences, dans D. Delattre \& J. Pigeaud 2010 (dir.), p. 535-562.

BraEt, A. C. 2004 : « Hermagoras and the Epicheireme », Rhetorica : A Journal of the History of Rhetoric, 22/4 (2004), p. 327-347.

Bremmer, J. N. 2007 : «Atheism in Antiquity » dans M. Martin (éd.), The Cambridge Companion to Atheism, Cambridge, 2007, p. 11-26 (Cambridge Companions to Philosophy).

Brunschwig, J. 2007 (éd.) : Aristote, Topiques, Tome II, Livres V-VIII, Paris, 2007 (Collection des universités de France. Série grecque, 451).

Delattre, D. \& J. Pigeaud 2010 (dir.) : Les Epicuriens, Paris, 2010 (Bibliothèque de la Pléiade, 564).

De Falco, V. 1923 (éd.) : L’Epicureo Demetrio Lacone, Napoli, 1923 (Biblioteca di filologia classica, 2).

De Lacy, P. H. \& E. A. De Lacy 1941 (éd.) : Philodemus, On Method of Inference: A Study in Ancient Empiricism, Philadelphia, 1941 (Philological Monographs, 10).

DoRANDI, T. 1999 (trad.) : Diogène Laërce, Vies et doctrines des philosophes illustres, Livre IV, Vie de Bion (46-58), dans M.-O. Goulet-Cazé (dir.) 1999, p. 525-531.

Dorandi, T. \& D. Delattre 2010 (trad.) : Démétrius Lacon, [La forme du dieu], dans D. Delattre \& J. Pigeaud 2010 (dir.), p. 257-260.

Drachmann, A. B. 1922 : Atheism in Pagan Antiquity, London, 1922.

Durkheim, E. 1990 [1912] : Les Formes élémentaires de la vie religieuse : le système totémique en Australie, Paris, 1990 [1912] (Quadrige, 77).

Festugière, A. J. 1946 : Épicure et ses dieux, Paris, 1946 (Mythes et religions, 19).

Foucault, M. 2009 : Le Courage de la vérité: Le gouvernement de soi et des autres, cours au Collège de France 1983-1984, II, édition établie par F. Gros, sous la direction de F. Ewald et A. Fontana, Paris, Gallimard-Seuil, 2009 (Hautes études).

Fuentes GonzÁlez, P. P. 1998 (éd.) : Les diatribes de Télès : introduction, texte revu et commentaire des fragments, avec en appendice une traduction espagnole, préf. de M.-O. Goulet-Cazé, Paris, 1998 (Histoire des Doctrines de l'Antiquité Classique, 23).

GERNET, L. 1917 : Recherches sur le développement de la pensée juridique et morale en Grèce : étude sémantique, Paris, 1917 (Bibliothèque de la Fondation Thiers, 37).

Giannantoni, G. 1990 (éd.) : Socratis et Socraticorum Reliquiae, Napoli, 1990 (Elenchos, 18).

Gigante, M. \& G. Indelli 1978 : « Bione e l'epicureismo », Cronache Ercolanesi, 8 (1978), p. 124-131.

Goulet-Cazé, M.-O. 1993 : «Les premiers Cyniques et la religion », dans M.-O. Goulet-Cazé et R. Goulet (éd.), Le Cynisme ancien et ses prolongements : 
actes du colloque international du CNRS, Paris, 22-25 juillet 1991, Paris, 1993, p. 117-158.

- 1999 (dir.) : Diogène Laërce, Vies et doctrines des philosophes illustres, Paris, 1999 (La Pochothèque).

Husson, S. 2011 : La République de Diogène : une cité en quête de la nature, Paris, 2011 (Histoire des doctrines de l'Antiquité classique, 40).

Kindstrand, J. F. 1976 (éd.) : Bion of Borysthenes, A Collection of the Fragments, Stockholm, 1976 (Studia Graeca Upsaliensia, 11).

Long, A. A. \& D. Sedley 1987 (éd.) : The Hellenistic Philosophers, vol. 1, Translations of the principal sources with philosophical commentary, Cambridge, 1987.

Mansfeld, J. 1993 : « Aspects of Epicurean Theology », Mnemosyne, $4^{\text {th }}$ Series, 46/2 (1993), p. 172-210.

Martin, D. 2004 : Inventing Superstition : from the Hippocratics to the Christians, Cambridge, 2004.

Mugler, C. 1956 : «L'isonomie des atomistes », Revue de Philologie, de Littérature et d'Histoire Anciennes, 30 (1956), p. 231-250.

Navia, L. E. 1996 : Classical Cynicism : a Critical Study, Wesport (Conn.), 1996 (Contributions in Philosophy, 58).

Préchac, F. 1927 (éd.) : Sénèque, Des bienfaits, t. II, Paris, 1927.

Santoro, M. 2000 (éd.) : Demetrio Lacone, La forma del dio : PHerc. 1055, ed., trad. e com, Napoli, 2000 (La scuola di Epicuro, 17).

Sorel, R. 2015 : Dictionnaire dupaganisme grec: notions et débats autour de l'époque classique, préf. de J.-F. Mattéi, Paris, 2015 (Vérité des mythes, 44).

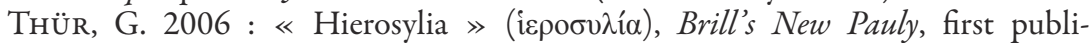
shed online: 2006, consulted online on 01 December 2017 http://dx.doi. org/10.1163/1574-9347_bnp_e513470.

VEYNE, P. 2000 : « Inviter les dieux, sacrifier, banqueter : quelques nuances de la religiosité gréco-romaine », Annales. Histoire, Sciences Sociales, 55/1 (2000), p. 3-42.

Villey, P. 1988 (éd.) : Montaigne, Les Essais, avec appendices, sources, index, éd. conforme au texte de l'exemplaire de Bordeaux, préf. par V. L. Saulnier, Paris, 1988 (Quadrige).

WiniarczyK, M. 1976 : «Der erste Atheistenkatalog des Kleitomachos », Philologus, 120/1 (1976), p. 32-46.

- 1981a (éd.) : Diagorae Melii et Theodori Cyrenaei Reliquiae, Leipzig, 1981a

(Bibliotheca scriptorum graecorum et romanorum Teubneriana. Auctores graeci).

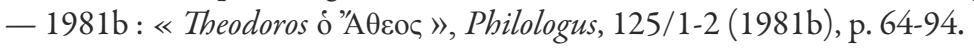

